\title{
Depresión, ansiedad e ira en adultos que sufrieron ataque al miocardio: un estudio de caso-control ${ }^{1}$
}

\author{
Zoilo Emilio García-Batista ${ }^{2}$, Kiero Guerra-Peña ${ }^{3}$, Antonio Cano-Vindel ${ }^{4}$, \\ Solmary Xiomara Herrera-Martínez ${ }^{5}$, Pablo Ezequiel Flores-Kanter ${ }^{6}$, \\ Leonardo Adrián Medrano ${ }^{7}$ \\ Pontificia Universidad Católica Madre y Maestra-República Dominicana ${ }^{2,3}$, \\ Universidad Complutense de Madrid- España ${ }^{4}$, Universidad Arturo Michelena- \\ Venezuela ${ }^{5}$ Universidad Siglo 21-Argentina ${ }^{6,7}$, Universidad Nacional de Córdoba- \\ Argentina $^{7}$
}

Las Enfermedades Cardiovasculares (ECV) son unas de las principales causas de mortalidad a nivel mundial. Diversos estudios han propuesto que los factores psicológicos explican la morbilidad y mortalidad de las ECV tanto a nivel primario como secundario. La presente investigación tuvo como principal objetivo verificar los niveles de depresión, ansiedad e ira en personas que han sufrido ataque de miocardio. Mediante un diseńo de caso-control, se contrastaron dos grupos, un grupo conformado por personas que habían padecido un ataque al miocardio (ECV, $n=33$ ), y otro grupo que sirvió de control equiparado en edad y sexo $(n=33)$. Si bien todas las variables contrastadas presentan niveles más altos en el grupo $\mathrm{ECV}$, solo la depresión presenta una diferencia estadísticamente significativa.

Palabras clave: depresión, ansiedad, ira, enfermedad cardiovascular, comorbilidad.

\section{Depression, Anxiety and Anger in Adults Who Suffered Myocardial Infarction: A Case- Control Study}

Cardiovascular Diseases (CVD) are one of the main causes of mortality worldwide. Several studies have proposed that psychological factors explain the morbidity and mortality of CVD, both at the primary and secondary levels. The main objective of the present investigation was to verify the levels of depression, anxiety and anger in people who have suffered a myocardial infarction. Using a case-control design, two groups were contrasted, a group consisting of people who had suffered a myocardial infarction (CVD, $n=33$ ), and another

1 Esta investigación contó con el apoyo del Fondo Nacional de Innovación y Desarrollo Científico y Tecnológico (FONDOCYT) de la República Dominicana. El primer y segundo autor reconocen grandemente a MESCyT y PUCMM por su sólido apoyo financiero, administrativo y técnico.

2 Doctor en Cognición Emoción y Estrés. Docente Escuela de Psicología. Pontifica Universidad Católica Madre y Maestra, Autopista Duarte Km 1 1/2, Santiago de los Caballeros, República Dominicana. Código postal 51000. Contacto: zgarcia@pucmm.edu.do. http:// orcid.org/0000-0002-0353-4804

3 Doctor en Métodos Cuantitativos. Docente Escuela de Psicología. Pontifica Universidad Católica Madre y Maestra, Autopista Duarte Km 1 1/2, Santiago de los Caballeros, República Dominicana. Código postal 51000. Contacto: kpena@pucmm.edu.do. https://orcid.org/ 0000-0003-3315-9459. 
group that served as a control matched by age and sex $(n=33)$. Although all the contrasted variables present higher levels in the CVD group, only depression presents a statistically significant difference.

Keywords: Depression, anxiety, anger, cardiovascular disease, comorbidity.

\section{Depressáo, ansiedade e raiva em adultos que sofreram ataque miocárdico: um estudo de caso-controle}

As Doenças Cardiovasculares (DCV) são uma das principais causas de mortalidade no mundo. Diversos estudos têm proposto que fatores psicológicos explicam a morbimortalidade das DCV tanto no nível primário como no secundário. O principal objetivo desta pesquisa foi verificar os níveis de depressão, ansiedade e raiva em pessoas que sofreram um ataque miocárdico. Usando um desenho de caso-controle, dois grupos foram contrastados, um grupo de pessoas que sofreram um ataque miocárdio (DCV, $n=33$ ), e outro grupo que serviu como controle pareado em idade e sexo $(n=33)$. Apesar de todas as variáveis contrastadas apresentarem níveis mais elevados no grupo DCV, apenas a depressão apresenta diferença estatisticamente significante.

Palavras-chave: depressão, ansiedade, ira, doença cardiovascular, comorbidade.

\section{Dépression, anxiété et colère chez les adultes ayant subi une attaque du myocarde: Une étude cas-témoins}

Les maladies cardiovasculaires (MCV) sont l'une des principales causes de mortalité dans le monde. Plusieurs études ont proposé que des facteurs psychologiques expliquent la morbidité et la mortalité des MCV, tant au niveau primaire que secondaire. L'objectif principal de la présente enquête était de vérifier les niveaux de dépression, d'anxiété et de colère chez les personnes ayant subi une attaque du myocarde. En utilisant un modèle de contrôle de cas, deux groupes ont été mis en contraste, un groupe constitué de personnes ayant subi une attaque du myocarde $(\mathrm{MCV}, \mathrm{n}=33)$ et un autre groupe ayant servi de groupe témoin en âge et en sexe $(n=33)$. Bien que toutes les variables contrastées présentent des niveaux plus élevés dans le groupe des maladies cardiovasculaires, seule la dépression présente une différence statistiquement significative

Mots-clés: dépression, anxiété, ira, maladie cardiovasculaire, comorbidité.

4 Doctor en Cognición, Emoción y Estrés. Docente Facultad de Psicología. Universidad Complutense de Madrid, Av. Séneca, 2, Madrid, España. Código postal 28223. Contacto: acanovin@ucm.es. https://orcid.org/0000-0002-5449-5454

5 Licenciada en Psicología. Docente e investigadora. Universidad Arturo Michelena, Av. Principal Giovanni Nani, a $1 \mathrm{Km}$. del Distribuidor La Cumaca, Sector El Polvero, San Diego 2006, Carabobo, Venezuela. Código postal 2040. Contacto: sohema55@hotmail.com.

6 Doctorando en Psicología. Docente e Investigador en Universidad Siglo 21, Calle de Los Latinos 8555, Córdoba, Argentina. Código postal 5000. Contacto: ezequielfk@gmail. com. http://orcid.org/0000-0002-6712-779X.

7 Doctor en Psicología. Docente e investigador. Universidad Siglo 21, Calle de Los Latinos 8555, Córdoba, Argentina. Código postal 5000. Pontifica Universidad Católica Madre y Maestra, Autopista Duarte Km 1 1/2, Santiago de los Caballeros, República Dominicana. Código postal 51000. Contacto: leomedpsico@gmail.com. http://orcid.org/0000-0002-3371-5040. 
Dentro de las enfermedades no comunicables o crónicas (Noncommunicable diseases, NCD, por sus siglas en inglés), los desórdenes del corazón y los vasos sanguíneos (i.e. Enfermedades Cardiovasculares, ECV) se identifican como uno de las más prevalentes (World Health Organization, 2018), posicionándose además como unas de las principales causas de mortalidad a nivel mundial (Kahl, Stapel \& Frieling, 2019; Stoney, Kaufmann \& Czajkowski, 2018; Vos, et al., 2017). Diversos estudios han propuesto que los factores psicológicos explican la morbilidad y mortalidad de las ECV tanto a nivel primario como secundario (Kollia, et al., 2017; Mathur, Foguet-Boreu, Ayis \& Ayerbe, 2016; Stoney et al., 2018), y se encuentran a la par del riesgo conferido a factores clásicos como el cigarrillo, la mala alimentación, y la inactividad física (Gianaros \& Jennings, 2018). Entre los factores psicológicos de riesgo, la depresión, la ansiedad y la ira han sido de las variables más frecuentemente estudiadas.

La mayor parte de la literatura científica sobre el tema se ha centrado en indagar cómo estas variables afectivas predicen las ECV (Gianaros \& Jennings, 2018). Respecto a esto, la evidencia no es homogénea. Mientras que para la depresión existe evidencia consistente en cuanto a su asociación con las ECV (Penninx, 2016; Suls \& Bunde, 2005; Xu et al., 2018), se han encontrado resultados dispares para el caso de la ansiedad y la ira (Emdin et al., 2016; Kupats et al., 2018; Mathur et al., 2016; Tully, 2017). Más aún, en el caso de la ira, la evidencia sugeriría que no existe una vinculación con las ECV. Esta es muy débil, o incluso presenta una relación negativa (Nakamura et al., 2013; Suls, 2013; Suls \& Bunde, 2005).

Sin embargo, recientemente se ha empezado a hacer hincapié en el estudio de la afectividad negativa una vez que se presenta la ECV (Chauvet-Gélinier, Trojak, Vergés-Patois, Cottin \& Bonin, 2013; Davidson, Alcántara \& Miller, 2018; Dornelas \& Sears, 2018; Doyle, 
et al., 2015). Por ejemplo, desde estas líneas de investigación se propone que la depresión no solo debe ser vista como un factor de riesgo para padecer un trastorno cardiovascular, sino que, además, la sintomatología depresiva puede presentarse una vez padecida la ECV empeorando el cuadro posterior (Doyle, et al., 2015; Penninx, 2016; Stoney et al., 2018; Zhang, Chen \& Ma, 2018). Aun así, la indagación sobre esta comorbilidad una vez presentada la enfermedad cardiovascular es reciente y se ha centrado principalmente en la depresión (Pedersen, von Kanel, Tully \& Denollet, 2017).

Debido a lo anterior, la presente investigación tiene como principal objetivo analizar los niveles de depresión, ansiedad e ira en personas que han sufrido ataque de miocardio. Este estudio es relevante dado que, por un lado, permitirá obtener evidencia en una población hasta ahora no estudiada, considerando participantes de República Dominicana. Más aún, si bien existe un único antecedente que ha indagado variables similares en personas con patología isquémica en Colombia (Lemos \& Agudelo-Vélez, 2018), su objetivo estuvo ligado a validar un modelo bifactor de afectividad negativa, y no el análisis o identificación de la sintomatología emocional característica en este grupo de pacientes comprándolo con un grupo control. En este marco, investigaciones previas han hecho hincapié en la necesidad de obtener mayor evidencia de la relación propuesta en las regiones de Latinoamérica y el Caribe (Camacho, Echeverría, Barros, Maiz \& Rigotti, 2018). Además, se propone dar a conocer la sintomatología más característica en este grupo de personas con ECV. Este último aspecto ha sido descuidado por las investigaciones previas, desconociéndose hasta el momento aquellos síntomas afectivos específicos que son más comunes en personas que han sufrido un ataque de miocardio. Finalmente, recientemente Klakk et al., (2018) han seńalado la necesidad de incluir en estos estudios la población de adultos jóvenes, la cual ha sido escasamente considerada en los antecedentes, y será objeto de investigación de este trabajo. 


\section{Método}

\section{Participantes}

Participaron en el presente estudio un total de 66 adultos seleccionados por medio de un muestreo no probabilístico. Para definir la categoría adultos se siguieron los criterios internacionales (Ruiz, 2005; Vos, et al., 2017). Según estos criterios, pueden considerarse como adultos a las personas en el rango etario de 20 a 60 años. Se contrastaron dos grupos, un grupo conformado por personas que habían padecido un ataque al miocardio, el Grupo ECV ( $\mathrm{n}=33)$, y otro grupo que sirvió de control equiparado en edad y sexo, el Grupo Control $(n=33)$. En la tabla 1 se especifican los datos sociodemográficos de ambos grupos, así como las similitudes en cuanto a edad y sexo.

\section{Medición}

Inventario de Ansiedad Estado/Rasgo (STAI). Este cuestionario evalúa la ansiedad estado y la ansiedad rasgo mediante 20 reactivos para cada una, con una escala de res-puesta tipo Likert de 4 alternativas. Para el presente estudio se aplicó la versión validada en la población dominicana. En dicha población las puntuaciones presentaron adecuadas propiedades psicométricas ( $\alpha$ entre .83 y .86), y relaciones significativas con la depresión (García-Batista, Guerra-Peña, Cano-Vindel, HerreraMartínez, Flores-Kanter \& Medrano, 2017). Además, se consideró solamente la medida de Ansiedad Rasgo.

Inventario de Expresión de Ira Rasgo-Estado (STAXI-2). El STAXI-2 ofrece una evaluación que diferencia los distintos elementos de la ira: experiencia, expresión y control, así como también de sus facetas tanto de Estado como de Rasgo. El STAXI-2 está compuesto por 49 elementos organizados en 6 escalas y 5 subescalas y posibilita obtener un índice de cada escala y sub-escala. Aquí también se utilizó la versión validada en República Dominicana. Los resultados dan cuenta de una aceptable y óptima consistencia interna ( $\alpha$ entre .75 y .86) y diferencias significativas entre población general y clínica (García-Batista, Guerra-Peña, 
Cano-Vindel, Herrera-Martínez, Flores-Kanter \& Medrano, 2018). En este caso también se consideró solamente la medida de rasgo de ira.

Inventario de depresión de Beck II (BDI-II; Beck, Steer \& Brown, 1996). Instrumento de autoinforme basado en los síntomas descritos por el Manual Diagnóstico y Estadístico de los Desórdenes Mentales (DSM-IV; American Psychiatric Association, 2000), que posibilita la medición de la severidad depresiva. La versión utilizada del inventario consiste en 21 ítems, en los cuales se presentan 4 opciones de respuesta en una escala de 0 a 3. En el presente estudio se utilizó la versión validada del BDI-II para a la población dominicana (GarcíaBatista, Guerra-Peña, Cano-Vindel, Herrera-Martínez \& Medrano, 2018), que permite medir de manera general la severidad depresiva, y también los subcomponentes cognitivos, afectivos y somáticos de la depresión. Más específicamente la validación dominicana permitió constatar la adecuación de un modelo bifactor $(\chi 2=541.57$; CFI=.94; RMSEA $=.05)$ y valores de consistencia interna óptima para la dimensión general $(\alpha=.89)$ y aceptables para las subdimensiones específicas (valores $\alpha$ de Cronbach entre .70 y .78).

\section{Procedimiento}

La investigación sigue un diseño de caso-control (Woodward, 2014). El grupo de personas con trastornos cardiovasculares fue seleccionado en las consultas del Hospital José María Cabral y Báez, localizado en la ciudad de Santiago de Los Caballeros, República Dominicana. Los doctores especialistas en cardiología diagnosticaban las personas con trastornos cardiovasculares y luego eran referidos al equipo de investigadores. En esta fase se les explicó de forma clara y breve los objetivos de la investigación, asegurando su participación voluntaria y anónima. Obtenido el consentimiento verbal, se tomaron los datos del paciente aplicando además los instrumentos STAI, STAXI-2 y BDI-2.

Posteriormente se seleccionó una muestra de la población general de República Dominicana, sin problemas coronarios diagnosticados, buscando que la misma tenga similares características en cuanto a cantidad de casos, sexo, y edad que el grupo de ECV. Una vez que estos 
accedían, se les explicó también de forma clara y breve los objetivos de la investigación, asegurando su participación voluntaria y anónima. Obtenido el consentimiento se aplicaron las pruebas correspondientes (i.e. STAI, STAXI-2 y BDI-2).

\section{Análisis de datos}

Para el análisis de los datos se utilizó principalmente la prueba $t$ para muestras independiente, dado el objetivo de contrastar dos grupos. Adicionalmente para verificar la magnitud de las diferencias se calculó el tamaño del efecto mediante la d de Cohen. Lo anterior resulta relevante dado que permite no solo basar las conclusiones e implicancias de una investigación en la significación estadística sino también considerando la significación práctica (i.e. magnitud del efecto) de los resultados (Lakens, 2013). Los análisis fueron efectuados con el software estadístico IBM SPSS 20.

\section{Resultados}

En primer lugar, se verificó que los grupos estuvieran equilibrados en cuanto al sexo y la edad (tabla 1). Para esto se constató que no hubiera diferencias estadísticamente significativas en las variables mencionadas. Los grupos no difieren en cuanto a sus principales variables sociodemográficas.

\section{Tabla 1}

Variables sociodemográficas: comparación respecto a la edad y el sexo

\begin{tabular}{llccccc}
\hline & & Grupo ECV & Grupo C. & $t$ & $X^{2}$ & $p$ \\
\hline Edad & M (DE) & $46.52(12.22)$ & $46.33(12.11)$ & .061 & & .952 \\
Sexo & Varones & $60.6 \%$ & $60.6 \%$ & & & \\
& Mujeres & $39.4 \%$ & $39.4 \%$ & & .001 & .999 \\
\hline
\end{tabular}

Nota. DE=Desviación Estándar; Grupo C.=Grupo Control. 
A continuación, se verificó si los grupos diferían en los niveles generales de depresión, rasgo de ira y ansiedad (tabla 2). Si bien todas las variables presentan niveles más altos en el Grupo de ECV, solo la depresión en su dimensión general $(t=2.33, p=.02, d=.58)$, afectiva $(t=2.11, p=.04, d=.53)$ y somática $(t=2.59, p=.01, d=.65)$ presenta una diferencia estadísticamente significativa. La diferencia más grande entre los grupos se observa en el factor somático de depresión $(d=.65)$. Para controlar la influencia del tamaño muestral y la forma de distribución de las variables consideradas (Wilcox \& Keselman, 2003), se repitió el análisis mediante la prueba no paramétrica U de Mann-Whitney. Los resultados no se vieron modificados (por razones de espacio sólo se muestran los análisis con la prueba $\mathrm{t}$ ).

\section{Tabla 2}

Niveles generales de depresión, rasgo de ansiedad e ira, entre el Grupo ECV y el Grupo Control

\begin{tabular}{|c|c|c|c|c|c|}
\hline & \multicolumn{2}{|c|}{ Grupos } & \multirow[b]{2}{*}{$t$} & \multirow[b]{2}{*}{$p$} & \multirow[b]{2}{*}{$d$} \\
\hline & $\begin{array}{c}\text { Grupo ECV } \\
M(D E)\end{array}$ & $\begin{array}{c}\text { Grupo C. } \\
M(D E)\end{array}$ & & & \\
\hline Depresión & $10.81(8.68)$ & $6.45(6.35)$ & 2.33 & .02 & .58 \\
\hline Cognitivo & $2.51(3.17)$ & $1.63(2.31)$ & 1.28 & .20 & .32 \\
\hline Afectivo & $2.15(2.41)$ & $1.09(1.56)$ & 2.11 & .04 & .53 \\
\hline Somático & $6.15(4.28)$ & $3.72(3.22)$ & 2.59 & .01 & .65 \\
\hline Rasgo de ansiedad & $14.48(7.98)$ & $11.21(7.80)$ & 1.68 & .09 & .42 \\
\hline Rasgo de ira & $16.93(6.18)$ & $16.03(6.23)$ & .59 & .55 & .15 \\
\hline Temperamento & $7.63(3.72)$ & $7.24(3.09)$ & .46 & .64 & .12 \\
\hline Reacción & $9.30(3.44)$ & $8.78(3.57)$ & .60 & .55 & .15 \\
\hline
\end{tabular}

Nota. Se resalta con el formato negrita aquellas variables que presentan diferencias estadísticamente significativas. M=Media; DE=Desviación Estándar; Grupo C.=Grupo Control.

Finalmente se analizaron las diferencias en la depresión a nivel de los síntomas (tabla 3). La tabla 3 permite apreciar que los síntomas 
más característicos del Grupo ECV se corresponden con pérdida de placer $(d=.68)$, llanto $(d=.65)$, agitación $(d=.57)$ y pérdida de interés sexual $(d=.54)$. En todos estos síntomas las diferencias evidenciadas presentan una magnitud del efecto moderada. En este caso también se repitió el análisis mediante la prueba no paramétrica U de MannWhitney. Los resultados tampoco se vieron modificados (por razones de espacio solo se muestran los análisis con la prueba t).

\section{Tabla 3}

Niveles de sintomatología depresiva y ansiógena: Comparación entre Grupo ECV y Grupo Control

\begin{tabular}{lccccc}
\hline & Grupo ECV & Grupo C. & & & \\
& $M(D E)$ & $M(D E)$ & $t$ & $p$ & $d$ \\
\hline Tristeza & $.48(.90)$ & $.18(.52)$ & 1.66 & .10 & .42 \\
Pesimismo & $.12(.54)$ & $.15(.36)$ & -.26 & .79 & .07 \\
Fracaso & $.09(.38)$ & $.18(.58)$ & -.74 & .46 & .19 \\
Pérdida de placer & $.88(1.02)$ & $.33(.54)$ & 2.70 & .01 & .68 \\
S. de culpa & $.48(.79)$ & $.33(.64)$ & .85 & .40 & .21 \\
S. de castigo & $.54(1.00)$ & $.33(.81)$ & .94 & .35 & .24 \\
Disconformidad & $.24(.56)$ & $.18(.46)$ & .48 & .63 & .12 \\
Autocrítica & $.57(.93)$ & $.39(.55)$ & .95 & .34 & .24 \\
Pensamiento S. & $.09(.29)$ & $.09(.38)$ & .01 & .99 & .01 \\
Llanto & $.78(1.08)$ & $.21(.64)$ & 2.61 & .01 & .65 \\
Agitación & $.78(.85)$ & $.36(.65)$ & 2.26 & .03 & .57 \\
Perdida de interés & $.57(.66)$ & $.33(.64)$ & 1.50 & .13 & .38 \\
Indecisión & $.36(.78)$ & $.12(.33)$ & 1.63 & .10 & .41 \\
Desvalorización & $.21(.69)$ & $.09(.38)$ & .87 & .38 & .22 \\
Perdida de energía & $.81(.91)$ & $.60(.55)$ & 1.13 & .26 & .28 \\
Cambios en H.S. & $.69(.76)$ & $.60(.70)$ & .50 & .61 & .13 \\
Irritabilidad & $.36(.60)$ & $.27(.67)$ & .57 & .56 & .14 \\
Cambios de apetito & $.51(.71)$ & $.30(.52)$ & 1.37 & .17 & .34
\end{tabular}




\begin{tabular}{lccccc}
\hline & Grupo ECV & Grupo C. & & & \\
& $M(D E)$ & $M(D E)$ & $t$ & $p$ & $d$ \\
\hline Dificultad en C. & $.60(.82)$ & $.45(.61)$ & .84 & .40 & .21 \\
Fatiga & $.93(.89)$ & $.66(.59)$ & 1.45 & .15 & .36 \\
Pérdida de I.S. & $.63(.85)$ & $.24(.61)$ & 2.14 & .04 & .54 \\
\hline
\end{tabular}

Nota. Se resalta con el formato negrita aquellas variables que presentan diferencias estadísticamente significativas. M=Media; DE=Desviación Estándar; Grupo C.=Grupo Control; S. de Culpa $=$ Sentimiento de Culpa; $S$. de Castigo=Sentimiento de Castigo; Pensamiento S.=Pensamiento Suicida; Cambios en H.S.=Cambios en Hábitos de Sueño; Dificultad en C.=Dificultad en la Concentración; Pérdida de I.S.=Pérdida de Interés Sexual.

\section{Discusión}

La presente investigación se propuso como principales objetivos a) verificar los niveles de depresión, ansiedad e ira en personas que habían padecido un accidente cardiovascular, y b) identificar los síntomas más característicos respecto a estas variables en las personas que padecen de ECV.

Respecto al primer objetivo, si bien en todas las variables se observan mayores niveles en el grupo ECV, splo en el caso de la depresión se observaron diferencias estadísticamente significativas, y de moderada magnitud, en favor de aquellas personas que habían padecido ataque al miocardio. En cuanto al segundo objetivo, los síntomas más característicos de las personas con ECV refieren a la pérdida de placer, el llanto, la agitación y la pérdida de interés sexual. Estos resultados son coincidentes con los recientes metaanálisis y estudios epidemiológicos que indican que la depresión y sus síntomas son frecuentes en personas que han padecido una ECV (Davidson, et al., 2018; Doyle, et al., 2015; Pedersen, et al., 2017; Zhang, et al., 2018). Además, los resultados coinciden también con el metaanálisis recientemente publicado por Le, Dorstyn, Mpfou, Prior y Tully (2018), en donde se evidencia que los sentimientos de fatiga y el estado anímico decaído son síntomas que surgen como consecuencia directa o indirecta de la enfermedad cardiovascular. 
Cabe destacar que los niveles de depresión obtenidos por el grupo de ECV, a pesar de presentar puntajes más altos en comparación al grupo control, pueden considerarse relativamente bajos (si se los compara, por ejemplo, con el puntaje máximo de 63 que tiene la escala total). Algunos antecedentes previos han verificado que la prevalencia de depresión, verificada mediante el BDI-II, es más alta en los niveles bajos que altos de la escala en este grupo de pacientes. Por ejemplo, Desai, et al. (2018), constataron que la prevalencia de los niveles de depresión en 250 pacientes con ECV se distribuyó de la siguiente manera: Normal (puntajes entre 0-10) $=68 \%$, Trastorno Leve (puntaje $>10 \mathrm{y}<17$ ) $=15 \%$, Depresión moderada (puntaje $\geq 17$ $y<30)=15 \%$, Depresión Severa (puntaje entre 30-40) = 2\%, Extrema (puntaje $>40)=0 \%$. Estos datos son similares a los obtenidos por Moryś, Bellwon, Adamczyk y Gruchała (2015) en una población diferente. Es posible que los niveles síntomas afectivos varíen en función del lapso temporal que pasa entre el diagnóstico y el momento de las mediciones afectivas, de desórdenes emocionales o ansiedad (i.e. considerando la historia de la enfermedad coronaria, Chauvet-Gélinier \& Bonin, 2017). Otro factor poco considerado es la influencia que sobre estos niveles de depresión pueda tener los distintos tipos específicos de ECV, agrupados en base a su gravedad (Rafanelli, Offidani, Gostoli \& Roncuzzi, 2012). De todas maneras, en el caso del BDI-II, si bien el instrumento ha demostrado validez en su aplicación en población con problemas cardíacos, existe evidencia limitada acerca del uso de puntos de corte en personas con ECV, por lo cual la interpretación de estos resultados debe ser cautelosa (González-Roz, et al., 2019). Lo cierto es que, más allá de estos niveles apreciados, se ha observado de manera consistente que la presencia de depresión en pacientes con ECV (aún en niveles bajos y subclínicos) se asocia a una peor prognosis y recuperación, menor adherencia al tratamiento, y mayores rehospitalizaciones por eventos cardíacos (González-Roz, et al., 2019).

Desde un plano teórico, la asociación entre la depresión y la ECV debe entenderse de manera recursiva y no unidireccional (Davidson, et al., 2018; Zhang, et al., 2018). De este modo, la depresión no solo 
debe ser vista como un factor de riesgo para padecer un trastorno cardiovascular, sino que además la sintomatología depresiva puede presentarse una vez padecida la ECV empeorando el cuadro posterior (Allabadi, et al., 2019; Doyle, et al., 2015; Pedersen, et al., 2017; Penninx, 2016). Según Chauvet-Gélinier, et al., (2013), ante un evento cardiovascular la persona tenderá a presentar una respuesta psicológica que normalmente conlleva cierto decaimiento en el estado anímico, asociado con las posibles pérdidas que se perciban ligadas con la enfermedad. Si la persona no supera este estado inicial, con el paso del tiempo se presentará un auténtico estado depresivo. Esto a su vez empeorará el cuadro debido a dos mecanismos principales, uno conductual y otro biológico (Allabadi, et al., 2019; Davidson, et al., 2018; Pedersen, et al., 2017; Penninx, 2016). Desde el plano conductual, las personas depresivas tienden a presentar comportamientos de riesgo para la salud, aumentando la ingesta de alcohol y el consumo de cigarrillos, por ejemplo. En relación con esto, el presente estudio dio cuenta de que una de las sintomatologías depresivas que más distinguía al grupo con ECV era la pérdida de placer. Muchas de estas conductas nocivas para la salud han sido vinculadas en este sentido a la incapacidad para experimentar placer (i.e. anhedonia, falta de interés) asociándolas de este modo directamente con la depresión (e.g. Rao, 2006). Respecto a los mecanismos biológicos, se ha hecho hincapié en la desregulación del eje hipotálamo-pituitario-adrenal (HPA-axis) y su vinculación con la ECV (Chauvet-Gélinier \& Bonin, 2017; Gianaros \& Jennings, 2018). En favor de esta hipótesis, la presente investigación ha encontrado como sintomatología característica del grupo ECV la agitación, que podría estar dando cuenta de un estado de mayor activación simpática y menor activación parasimpática.

Los datos obtenidos por el presente trabajo evidencian la importancia de diagnosticar y tratar de forma temprana los síntomas depresivos en personas que hayan padecido un accidente cardiovascular (Davidson et al., 2018; Dornelas \& Sears, 2018). Existe evidencia de que el mejoramiento de los síntomas depresivos mejora la prognosis de la ECV (Pedersen, et al., 2017). En efecto, la resistencia al tratamiento 
de la depresión es un factor de riesgo para padecer nuevos accidentes cardiovasculares (Carney, et al., 2000; Chauvet-Gélinier, et al., 2013; Chauvet-Gélinier \& Bonin, 2017). Siguiendo esta línea, el tratamiento de los síntomas depresivos mediante terapia cognitiva conductual ha demostrado mejorar la sintomatología cardiovascular (Carney, et al., 2000). Sumado a lo anterior, la terapia cognitiva conductual ha demostrado ser eficaz para el manejo del estrés, lo cual resulta relevante en este grupo de personas en donde la reducción del estrés psicosocial se asocia un aumento en la expectativa de vida (Chauvet-Gélinier, et al., 2013). Dentro del abordaje cognitivo-conductual se ha hecho hincapié en intervenciones que promuevan la reinterpretación cognitiva, estando esta estrategia de regulación emocional vinculada con marcadores de riesgo de ECV (Gianaros \& Jennings, 2018). Intervenciones de tipo conductual han mostrado también evidencia de que el manejo del estrés reduce los factores de riesgos y eventos cardiovasculares (Blumenthal et al., 2005; 2016). Finalmente, el abordaje de estos factores psicológicos también ha demostrado ser relevante para aumentar la adherencia al tratamiento médico en este tipo de pacientes (Amico, Mugavero, Krousel-Wood, Bosworth \& Merlin, 2017; Bosworth, Blalock, Hoyle, Czajkowski \& Voils, 2018).

Es importante mencionar las limitaciones que ciertamente presenta este trabajo. Por un lado, el diseño implementado forma parte de los denominados diseños retrospectivos, los cuales no permiten sacar conclusiones definitivas respecto a la causalidad o relación temporal de las variables implicadas en el modelo. El paso de diseńos transaccionales a otros longitudinales permite a los investigadores hacer inferencias rigurosas sobre las relaciones causales implicadas en los modelos (Cole \& Maxwell, 2003). En este sentido, futuras investigaciones podrían testear el modelo mejorando el diseńo empleado, aplicando por ejemplo un diseño prospectivo de más de un eslabón causal (Montero \& León, 2006). También es sugerible incorporar en futuros estudios longitudinales medidas biológicas y fisiológicas referidas al funcionamiento cardíaco y vascular (Chauvet-Gélinier \& Bonin, 2017). 
No obstante lo anterior, la presente investigación presenta evidencia relevante, que da cuenta de la presencia de comorbilidad depresiva en personas que han padecido un accidente del miocardio. Dicha sintomatología depresiva, particularmente vinculada a las dimensiones afectiva y somática, debe ser foco de los profesionales de salud para el correcto abordaje médico/psicológico de estos pacientes.

\section{Referencias}

Allabadi, H., Alkaiyat, A., Alkhayyat, A., Hammoudi, A., Odeh, H., Shtayeh, J., ... Probst-Hensch, N. (2019). Depression and anxiety symptoms in cardiac patients: a cross-sectional hospitalbased study in a Palestinian population. BMC Public Health, 19(1). https://doi.org/10.1186/s12889-019-6561-3

American Psychiatric Association. (2000). Diagnostic and statistical manual of mental disorders (4th ed). Washington, DC: American Psychiatric Association.

Amico, K. R., Mugavero, M., Krousel-Wood, M. A., Bosworth, H. B. \& Merlin, J. S. (2017). Advantages to Using Social-Behavioral Models of Medication Adherence in Research and Practice. Journal of General Internal Medicine, 33(2), 207-215. https:// doi.org/10.1007/s11606-017-4197-5

Beck, A., Steer, R. \& Brown, G. (1996). Beck Depression Inventory. San Antonio, TX: Psychological Corporation. https://doi.org/ 10.1037/t00742-000

Bosworth, H. B., Blalock, D. V., Hoyle, R. H., Czajkowski, S. M. \& Voils, C. I. (2018). The role of psychological science in efforts to improve cardiovascular medication adherence. American Psychologist, 73(8), 968-980. https://doi.org/10.1037/amp0000316

Camacho, J., Echeverría, G., Barros, J., Maiz, A. \& Rigotti, A. (2018).

Depression and Stress are Highly Associated with Hypertension, Diabetes, and Cardiovascular Disease in Chilean Population. 
Atherosclerosis Supplements, 32, 74. https://doi.org/10.1016/j. atherosclerosissup.2018.04.226

Carney, R. M., Freedland, K., Stein, P. K., Skala, J. A., Hoffman, P. \& Jaffe, A. S. (2000). Change in heart rate and heart rate variability during treatment for depression in patients with coronary heart disease. Psychosomatic Medicine, 62, 639-647. https://doi. org/10.1097/00006842-200009000-00007

Chauvet-Gelinier, J.-C. \& Bonin, B. (2017). Stress, anxiety and depression in heart disease patients: A major challenge for cardiac rehabilitation. Annals of Physical and Rehabilitation Medicine, 60(1), 6-12. https://doi.org/10.1016/j.rehab.2016.09.002

Chauvet-Gélinier, J.-C., Trojak, B., Vergès-Patois, B., Cottin, Y. \& Bonin, B. (2013). Review on depression and coronary heart disease. Archives of Cardiovascular Diseases, 106(2), 103-110. https://doi.org/10.1016/j.acvd.2012.12.004

Cole, D. A. \& Maxwell, S. E. (2003). Testing Mediational Models With Longitudinal Data: Questions and Tips in the Use of Structural Equation Modeling. Journal of Abnormal Psychology, 112(4), 558-577. https://doi.org/10.1037/0021-843X.112.4.558

Davidson, K. W., Alcántara, C. \& Miller, G. E. (2018). Selected psychological comorbidities in coronary heart disease: Challenges and grand opportunities. American Psychologist, 73(8), 1019-1030. https://doi.org/10.1037/amp0000239

Desai, K., Diwan, N., Mudhiganti, P. D., Joshi, A. V., Boggula, N. \& Bakshi, V. (2018). Assessment of prevalence of depression in cardiac patients and its association with the use of beta-blockers and statins. Asian Journal of Pharmaceutical and Clinical Research, 11(12), 416. https://doi.org/10.22159/ajpcr.2018. v11i12.28444

Dornelas, E. A. \& Sears, S. F. (2018). Living with heart despite recurrent challenges: Psychological care for adults with advanced cardiac disease. American Psychologist, 73(8), 1007-1018. https://doi. org/10.1037/amp0000318 
Doyle, F., McGee, H., Conroy, R., Conradi, H. J., Meijer, A., Steeds, R., ... de Jonge, P. (2015). Systematic Review and Individual Patient Data Meta-Analysis of Sex Differences in Depression and Prognosis in Persons With Myocardial Infarction. Psychosomatic Medicine, 77(4), 419-428. https://doi.org/10.1097/PSY. 0000000000000174

Emdin, C. A., Odutayo, A., Wong, C. X., Tran, J., Hsiao, A. J. \& Hunn, B. H. M. (2016). Meta-Analysis of Anxiety as a Risk Factor for Cardiovascular Disease. The American Journal of Cardiology, 118(4), 511-519. https://doi.org/10.1016/j.amjcard. 2016.05.041

García-Batista, Z. E., Guerra-Peña, K., Cano-Vindel, A., HerreraMartínez, S. X. \& Medrano, L. A. (2018). Validity and reliability of the Beck Depression Inventory (BDI-II) in general and hospital population of Dominican Republic. PLOS ONE, 13(6), e0199750. https://doi.org/10.1371/journal.pone.0199750

García-Batista, Z. E., Guerra-Peña, K., Cano-Vindel, A., HerreraMartínez, S. X., Flores-Kanter, P. E. \& Medrano, L. A. (2018). Evidencias de validez y fiabilidad de las Puntuaciones del STAXI-2 para población general y hospitalaria: Estudio con una muestra de adultos de República Dominicana. Suma Psicológica, 25(1), 21-29. https://doi.org/10.14349/sumapsi.2018.v25.n1.3

García-Batista, Z. E., Guerra-Peña, K., Cano-Vindel, A., HerreraMartínez, S. X., Flores-Kanter, P. E. \& Medrano, L. A. (2017). Propiedades psicométricas del Inventario de ansiedad estado-rasgo en población general y hospitalaria de República Dominicana. Ansiedad y Estrés, 23(2-3), 53-58. https://doi. org/10.1016/j.anyes.2017.09.004

Gianaros, P. J. \& Jennings, J. R. (2018). Host in the machine: A neurobiological perspective on psychological stress and cardiovascular disease. American Psychologist, 73(8), 1031-1044. https://doi.org/10.1037/amp0000232

Kahl, K. G., Stapel, B. \& Frieling, H. (2019). Link between depression and cardiovascular diseases due to epigenomics and 
proteomics: Focus on energy metabolism. Progress in NeuroPsychopharmacology and Biological Psychiatry, 89, 146-157. https://doi.org/10.1016/j.pnpbp.2018.09.004

Klakk, H., Kristensen, P. L., Andersen, L. B., Froberg, K., Møller, N. C. \& Grøntved, A. (2018). Symptoms of depression in young adulthood is associated with unfavorable clinical- and behavioral cardiovascular disease risk factors. Preventive Medicine Reports, 11, 209-215. https://doi.org/10.1016/j.pmedr.2018.05.017

Kollia, N., Panagiotakos, D., Georgousopoulou, E., Chrysohoou, C., Yannakoulia, M., Stefanadis, C., ... Pitsavos, C. (2017). Exploring the path between depression, anxiety and 10-year cardiovascular disease incidence, among apparently healthy Greek middle-aged adults: The ATTICA study. Maturitas, 106, 73-79. https://doi.org/10.1016/j.maturitas.2017.09.005

Kupats, E., Noviks, I., Vrublevska, J., Kenina, V., Kojalo, U. \& Logina, I. (2018). No relationship between generalised anxiety symptoms and cardiovascular autonomic dysfunction. Neurology, Psychiatry and Brain Research, 30, 86-90. https://doi.org/10.1016/j.npbr. 2018.07.001

Lakens, D. (2013). Calculating and reporting effect sizes to facilitate cumulative science: a practical primer for t-tests and ANOVAs. Frontiers in Psychology, 4. https://doi.org/10.3389/ fpsyg.2013.00863

Le, J., Dorstyn, D. S., Mpfou, E., Prior, E. \& Tully, P. J. (2018). Healthrelated quality of life in coronary heart disease: a systematic review and meta-analysis mapped against the International Classification of Functioning, Disability and Health. Quality of Life Research, 27(10), 2491-2503. https://doi.org/10.1007/ s11136-018-1885-5

Lemos, M. \& Agudelo-Vélez, D. M. (2018). El afecto negativo como factor emocional de orden superior en pacientes cardiovasculares. Acta Colombiana de Psicología, 21(2), 68-87. https://doi. org/10.14718/ACP.2018.21.2.4 
Mathur, R., Pérez-Pinar, M., Foguet-Boreu, Q., Ayis, S. \& Ayerbe, L. (2016). Risk of incident cardiovascular events amongst individuals with anxiety and depression: A prospective cohort study in the east London primary care database. Journal of Affective Disorders, 206, 41-47. https://doi.org/10.1016/j.jad. 2016.07.046

Montero, I. \& León, O. (2006). A guide for naming research studies in Psychology. International Journal of Clinical and Health Psychology, 7(3), 847-862.

Moryś, J. M., Bellwon, J., Adamczyk, K. \& Gruchała, M. (2015).

Depression and anxiety in patients with coronary artery disease, measured by means of self-report measures and clinician-rated instrument. Kardiologia Polska, 74(1), 53-60. https:/doi. org/10.5603/KP.a2015.0116

Nakamura, S., Kato, K., Yoshida, A., Fukuma, N., Okumura, Y., Ito, H. \& Mizuno, K. (2013). Prognostic Value of Depression, Anxiety, and Anger in Hospitalized Cardiovascular Disease Patients for Predicting Adverse Cardiac Outcomes. The American Journal of Cardiology, 111(10), 1432-1436. https://doi.org/10.1016/ j.amjcard.2013.01.293

Pedersen, S. S., von Känel, R., Tully, P. J. \& Denollet, J. (2017). Psychosocial perspectives in cardiovascular disease. European Journal of Preventive Cardiology, 24(3), 108-115. https://doi. org/10.1177/2047487317703827

Penninx, B. W. J. H. (2017). Depression and cardiovascular disease: Epidemiological evidence on their linking mechanisms. Neuroscience \& Biobehavioral Reviews, 74, 277-286. https://doi. org/10.1016/j.neubiorev.2016.07.003

Rao, U. (2006). Links Between Depression and Substance Abuse in Adolescents. American Journal of Preventive Medicine, 31(6), 161-174. https://doi.org/10.1016/j.amepre.2006.07.002

Ruiz, J-F. M. (2005). Los factores definitorios de los grandes grupos de edad de la población: Tipos, subgrupos, umbrales. Revista Electrónica de Geografía y Ciencias Sociales, 9 (190). 
Stoney, C. M., Kaufmann, P. G. \& Czajkowski, S. M. (2018). Cardiovascular disease: Psychological, social, and behavioral influences: Introduction to the special issue. American Psychologist, 73(8), 949-954. https://doi.org/10.1037/amp0000359

Suls, J. (2013). Anger and the Heart: Perspectives on cardiac risk, mechanisms and interventions. Progress in Cardiovascular Diseases, 55, 538-547. https://doi.org/10.1016/j.pcad.2013.03.002

Suls, J. \& Bunde, J. (2005). Anger, Anxiety, and Depression as Risk Factors for Cardiovascular Disease: The Problems and Implications of Overlapping Affective Dispositions. Psychological Bulletin, 131(2), 260-300. https://doi.org/10.1037/0033-2909. 131.2.260

Tully, P. J. (2017). Anxiety and Incident Cardiovascular Disease: Is the Jury Still Out? The American Journal of Cardiology, 120(3), e21. https://doi.org/10.1016/j.amjcard.2016.06.027

Vos, T., Abajobir, A. A., Abate, K. H., Abbafati, C., Abbas, K. M., Abd-Allah, F., ... Abera, S. F. (2017). Global, regional, and national incidence, prevalence, and years lived with disability for 328 diseases and injuries for 195 countries, 1990-2016: a systematic analysis for the Global Burden of Disease Study 2016. The Lancet, 390(10100), 1211-1259. https://doi.org/10.1016/ S0140-6736(17)32154-2

Wilcox, R. R. \& Keselman, H. J. (2003). Modern Robust Data Analysis Methods: Measures of Central Tendency. Psychological Methods, 8(3), 254-274. https://doi.org/10.1037/1082-989X.8.3.254

Woodward, M. (2014). Epidemiology. Study Design and Data Analysis. Nueva York, NY: CRC Press. https://doi.org/10.1201/b16343 World Health Organization. (2018). Noncommunicable diseases country profiles 2018. Suiza: WHO Press.

Xu, M., Chen, R., Liu, B., Chai, Y., Boer, D. D., Hu, P. \& Hu, Z. (2018). Psychosocial determinants of depression in the community of the elderly with cardiovascular disease. Psychiatry Research, 268, 123-130. https://doi.org/10.1016/j.psychres.2018.03.008 
Zhang, Y., Chen, Y. \& Ma, L. (2018). Depression and cardiovascular disease in elderly: Current understanding. Journal of Clinical Neuroscience, 47,1-5. https://doi.org/10.1016/j.jocn.2017.09.022

Recibido: 9 de noviembre, 2018

Revisado: 29 de agosto, 2019

Aceptado. 12 de septiembre, 2019 